\title{
The use of multiplex-PCR method in identification of Candida species from vaginal candidiasis patients
}

\author{
SUSILAWATI ${ }^{1, \bullet}$, KEMAS YA'KUB RAHADIYANTO $^{1}{ }^{\prime}$ MUHAIMIN RAMDJA ${ }^{1}$, THEODORUS ${ }^{1}$, \\ HERMANSYAH ${ }^{2, v \bullet}$ \\ ${ }^{1}$ Program of Medicine, Faculty of Medicine, Universitas Sriwijaya. Jl. Dr. Moh. Ali, Komp. RSMH, Palembang 30126, South Sumatra, Indonesia. \\ Tel.: +62-711-373438, fax.: +62-711-580061, `email: susilwt78@gmail.com \\ ${ }^{2}$ Department of Chemistry, Faculty of Mathematics and Natural Sciences, Universitas Sriwijaya. Jl. Raya Palembang-Prabumulih Km 32, Indralaya, \\ Ogan Ilir 30662, South Sumatra, Indonesia. Tel.: +62-711-580269, Fax.: +62-711-580056, •vemail: hermansyah@unsri.ac.id
}

Manuscript received: 28 June 2019. Revision accepted: 28 September 2019.

\begin{abstract}
Susilawati, Rahadiyanto KY, Ramdja, Theodorus, Hermansyah. 2019. The use of multiplex-PCR method in identification of Candida species from vaginal candidiasis patients. Biodiversitas 20: 3063-3069. Vaginal Candidiasis is an infection caused by a yeast called Candida which may be resulted in different virulences, thus leading to a varied drug of choice for therapy. The objective of this study was to determine the sensitivity and specificity of multiplex-PCR test in detecting Candida species in women with clinical diagnosis of vaginal candidiasis. There were 79 vaginal swab samples which fulfill inclusion criteria. Samples were cultured at ASD media for isolation of Candida spp. Identification of Candida spp. was conducted using both biochemical fermentation and multiplexPCR methods. Primers pairs used in multiplex-PCR were universal primer ITS1 and ITS2, and specific primer CA3 and CA4. The identification test of multiplex-PCR resulted in Candida krusei: sensitivity: 100\%, specificity:61,1\%, Positive Prediction Value (PPV):63.2\%, Negative Prediction Value (NPV): 100\%; C. glabrata: sensitivity:0\%, specificity: 100\% PPV: 0\% NPV: $76.7 \%$;. tropicalis: sensitivity:100\%, spesificity:100\%, PPV: 100\% NPV: 100\%; C. albicans: sensitivity: 33.3\%, specificity: 100\%, PPV: 100\%, NPV: $93.1 \%$ and C. stellatoidea: sensitivity:: 100\%, specificity:92.6\%, PPV: 60\%, NPV: 100\%. These results suggested that multiplexPCR method becomes a rapid alternative in identifying species of Candida due to its high sensitivity and high specificity.
\end{abstract}

Keywords: Candida, candidiasis, multiplex-PCR, fermentation

Abbreviations: ASD: Agar Sabouraut Dextrose; PPV: Positive Prediction Value; NPV: Negative Prediction Value; C: Candida; PCR: Polymerase Chain Reaction

\section{INTRODUCTION}

The implication of Candida sp examination in reproductive canal has to pay more attention due to their harmful effects in the women like getting leukorrhea and itching which caused abrasions and uncomfortable sexual intercourse. Candidiasis vaginalis can also cause Candida chorioamnionitis that led to abortus or an infant born with congenital candidiasis, and moreover more $75 \%$ women had endured at least one episode of candidiasis vaginalis during her lifetime, and around half of them have candidiasis vaginalis recurring (Omar 2001). The cause of candidiasis vaginalis at several locations in Jakarta is Candida albicans $(62.3 \%)$, while non-Candida albicans is $30.4 \%$ which consists of Candida glabrata $18.8 \%$, Candida tropicalis $8.7 \%$, Candida parapsilosis $2.9 \%$ and mixed infection of total of $7.3 \%$ (Haryani et al. 2003). The isolation of fungi including Candida is generally undertaken by culturing the specimen on Sabouraud Dextrose Agar medium (SDA) who customarily used for isolation of various fungi. In this medium of all species of Candida grown as yeast colony or colonies such as yeasts that cannot be distinguished one another both a macroscopically and microscopically. Identification of species was done by test fermentation-assimilation and morphologically based conventional methods. During this method, fermentation-assimilation is regarded as raw gold and has been used widely and known to have high skill in identifying various species of Candida (Wahyuningsih et al 2012). An antibody-antigen specific test has been developed for identification of Candida spp., but proved low in sensitivity and specificity (Tarini et al. 2010). The success of treatment depends on the sensitivity of Candida species against anti-fungal. Species level identification is needed early in the case of candidiasis caused by Candida $\mathrm{sp}$ which is resistant to the azole so that alternative therapies can be immediately given. Conventional method (fermentation-assimilation) as the 'Gold Standard' has high sensitivity and specificity but require a long examination time (7-21 days) and troubles in terms of the provision of chemicals as well as the high costs. At this time many molecular tests that have been developed for identification Candida species and are being used due to their faster, more sensitive and more specific approach. One approach is molecular test multiplex-PCR which is the development, modification, and a variation of the method of analysis of the PCR in which two or more simultaneous amplified locus in the same reaction making it possible to have a high sensitivity and specification and low cost and also the fast result progress (Tarini et al. 2010). In the present study, 
research was conducted to assess the multiplex PCR's sensitivity and specificity in diagnosing the species of Candida on participants who allegedly suffer candidiasis vaginalis. The research design was the identification of Candida spp. by two different methods namely, multiplexPCR with biochemical test for fermentation as gold standard to compare their sensitivity and specificity.

\section{MATERIALS AND METHODS}

\section{Materials}

SDA medium consisted of $20 \mathrm{~g} / \mathrm{L}$ dextrose, $10 \mathrm{~g} / \mathrm{L}$ peptone, $17 \mathrm{~g} / \mathrm{L}$ bacto agar, and $250 \mathrm{mg} / \mathrm{L}$ chloramphenicol. Medium was sterilized in an autoclave at $121^{\circ} \mathrm{C}$ for $15 \mathrm{~min}$. Cellulose-acetate filter with a pore diameter $0.2 \mu \mathrm{m}$ yeast nitrogen base (YNB) medium (yeast nitrogen base), incubator, dextrose, galactose, sucrose, lactose, maltose and trehalose (all at a concentration of $6 \%$ ), melibiose, cellobiose, inositol, D-xylose and starch, raffinose were used in fermentation test.

\section{Samples of Candida sp.}

Samples of Candida spp. were isolated from vaginal swabs of all patients suffering with candidiasis vaginalis clinic diagnosed by Doctor in Graha Sriwijaya Clinic, Palembang, Indonesia. Swab samples were taken using sterilized cotton stick. To determine total sample used, formula used is given as: $\mathrm{N}=\{\mathrm{Z} \alpha 2 \mathrm{P}(1-\mathrm{P})\} / \mathrm{d} 2$, where $\mathrm{N}=$ the sample size, $Z \alpha=$ the $Z$ statistic for a $95 \%$ confidence level, $\mathrm{P}=$ sensitivity, and $\mathrm{d}=$ precision. It used sensitivity and specificity of Candida tropicalis which is $80 \%$ and $90.6 \%$, respectively which obtained in total sample of 68 peoples. Topical vaginal swab specimens were streaked in a zig-zag manner on ASD media, incubated at $30^{\circ} \mathrm{C}$ for $24-$ 48 hours. Then, color, shape, consistency, and size of the colonies of Candida spp. were observed. Positive colonies were indicated with creamy white color, rounded shape, curved, soft and smooth consistency form.

\section{Procedure}

\section{Biochemical fermentation}

Biochemical fermentation process was conducted according to (Wahyuningsih et al. 2012). Fungal suspension was prepared in vitro with $0.85 \% \mathrm{NaCl}$, which is derived from fungal colonies from SDA media. The suspension was homogenized with no clumps. Concentration of Candida spp. grown in the medium was adjusted to $5 \times 10^{6}$ cell $/ \mathrm{ml}$. Glucose, galactose, sucrose, lactose, maltose, and trehalose were used for filtration. Then incubation for 21 days at a temperature of $25^{\circ} \mathrm{C}$. When the color changed from green to yellow or if it changed color and form gas in Durham tubes indicated positive result, while if there was no color changed indicated negative result. Candida spp. determination was done by matching the fermentation pattern formed by reference.

\section{DNA preparation}

DNA extraction procedure was carried out according to protocol with small modification (da Silva et al. 2012). Cells were grown for 1 hour until obtained $10^{7}$ cells $/ \mathrm{mL}$ of Candida spp., transferred to microtubes, and incubated at boiling water-bath for $3 \times 5 \mathrm{~min}$. During interval time, the suspension containing DNA was vigorously homogenized by vortex for $5 \times 5$ seconds and the tube was frozen on ice. The DNA sample was stored at $-20^{\circ} \mathrm{C}$. DNA concentration was measured using Spectrophotometer (SmartSpec Plus) at $\lambda=260 \mathrm{~nm}$.

\section{Multiplex-PCR}

Analysis of multiplex PCR was carried out according to protocol with modification (Tarini et al. 2010). Primers pair used in this experiment were Universal primers ITS1: (5'-TCCGTAGGTGAACCTGCGG-3') and ITS2: (5'GCTGCGTTCTTCATCGATGC-3'), specific primary CA3: (5'-GGTTTGCTTGAAAGACGGTAG-3') and CA4: (5'-AGTTTGAAGATATACGTGGTAG-3'). multiplex PCR was performed with a total volume of $25 \mu \mathrm{L}$ consisting of $12.5 \mu \mathrm{L}$ dream Taq Green PCR Master mixt $2 \mathrm{x}$ (Thermo Science), $0.4 \mu \mathrm{M}$ each of ITS1 and ITS2 primers, $1.2 \mu \mathrm{M}$ each of $\mathrm{CA} 3$ and CA4 primers, 2-6 ng/reaction of DNA template. Products were amplified using the following condition pre-denaturation temperature at $95^{\circ} \mathrm{C}$ for 15 second, and, then 40 cycles at $94^{\circ} \mathrm{C}$ (denaturation temperature) for $30 \mathrm{sec}$, annealing temperature at $60^{\circ} \mathrm{C}$ for $60 \mathrm{sec}$, extension temperature at $72^{\circ} \mathrm{C}$ for $45 \mathrm{sec}$ followed by one cycle of $72^{\circ} \mathrm{C}$ for $5 \mathrm{~min}$. For detection of amplification results, The PCR products were analyzed by electrophoresis using $2 \%$ agarose gel at 100 volts for $50 \mathrm{~min}$. The DNA bands were detected by Gel doc 1000 with ultraviolet transilluminator (Biorad, USA), and visualized using Quantity One software (Biorad, USA). The length of products multiplex-PCR with primer pairs ITS1-ITS2 and CA3-CA4 detected by electrophoresis are: C. glabrata (482 bp 483 bp or 462 bp -463 bp), $C$. guillermondii (248 bp or $228 \mathrm{bp}$ ), C. parapsilosis (229 bp or $209 \mathrm{bp}$ ), C. tropicalis (218 bp or $199 \mathrm{bp}$ ), C. albicans (218 bp 219 bp or 198 bp -199 bp and 110 bp), C. krusei (182 bp or $166 \mathrm{bp}$ ), C. lusitaniae (148 bp or $128 \mathrm{bp}$ ), $C$. dubliniensis (198 bp), C. stellatoidea (190 bp) (Liguori et al. 2007, 2000).

\section{RESULTS AND DISCUSSION}

In this research, we used the total 30 samples, of which 28 peoples $(93.3 \%)$ were of $18-45$ year age group. The distribution data by age of 30 respondents can be seen in Table 1. The results were consistent with research conducted by Sobel et al. (2004) which showed the incidence of vaginal candidiasis is more common in young adult women (70-75\%) and rarely occurs in children and postmenopausal women who are not taking hormone replacement therapy. 
Table 1. Distribution of respondents by age $(n=30)$

\begin{tabular}{lcc}
\hline Age (Year) & Amount (person) & Percentage \\
\hline $18-45$ & 28 & 93.3 \\
$>45$ & 2 & 6.7 \\
Total & 30 & 100.0 \\
\hline
\end{tabular}

Table 2. Distribution of respondents by contraception user $(\mathrm{n}=$ 30)

\begin{tabular}{lcc}
\hline $\begin{array}{c}\text { Type of } \\
\text { contraception }\end{array}$ & $\begin{array}{c}\text { Amount } \\
\text { (person) }\end{array}$ & Percentage \\
\hline Pill & 18 & 60.0 \\
Injection & 6 & 20.0 \\
No contraception & 5 & 16.7 \\
Intra Uterine Device & 1 & 3.3 \\
Total & 30 & 100.0 \\
\hline
\end{tabular}

Table 3. Distribution of culture test results using ASD agar plate $(\mathrm{n}=79)$

\begin{tabular}{lcc}
\hline \multicolumn{1}{c}{ Culture } & Amount (person) & Percentage \\
\hline Positive & 30 & 38,0 \\
Negative & 49 & 62,0 \\
Amount & 79 & 100,0 \\
\hline
\end{tabular}

Table 4. Identification of Candida species with fermentation test and multiplex-PCR $(\mathrm{n}=30)$

\begin{tabular}{cll}
\hline No. sample & \multicolumn{1}{c}{ Fermentation } & \multicolumn{1}{c}{ Multiplex-PCR } \\
\hline 2 & C. tropicalis & C. tropicalis \\
4 & C. tropicalis & C. tropicalis \\
5 & C. stellatoidea & C. stellatoidea \\
6 & C. krusei & C. krusei, C. glabrata \\
7 & C. albicans & C. stellatoidea \\
12 & C. glabrata & C. krusei \\
13 & C. krusei & C. krusei \\
18 & C. glabrata & C. krusei \\
19 & C. krusei & C. krusei, C. glabrata \\
20 & C. stellatoidea & C. stellatoidea, C. glabrata \\
25 & C. krusei & C. krusei \\
31 & C. glabrata & C. krusei \\
32 & C. krusei & C. krusei \\
35 & C. glabrata & C. krusei \\
37 & C. tropicalis & C. tropicalis \\
39 & C. krusei & C. krusei \\
41 & C. tropicalis & C. tropicalis \\
44 & C. albicans & C. albicans \\
53 & C. krusei & C. krusei, C. glabrata \\
54 & C. glabrata & C. krusei \\
58 & C. glabrata & C. krusei \\
60 & C. krusei & C. krusei \\
61 & C. albicans & C. stellatoidea \\
63 & C. krusei & C. krusei, C. glabrata \\
65 & C. krusei & C. krusei \\
74 & C. glabrata & C. krusei \\
75 & C. krusei & C. krusei \\
77 & C. tropicalis & C. tropicalis \\
79 & C. krusei & C. krusei, C. glabrata \\
80 & C. stellatoidea & C. stellatoidea, C. glabrata \\
Amount & 30 & 30 \\
\hline & &
\end{tabular}

Table 5. Diagnostic test results $C$. tropicalis $(\mathrm{n}=30)$

\begin{tabular}{llccc} 
& & \multicolumn{3}{c}{ Fermentation test } \\
\cline { 3 - 5 } & & Positive & Negative & Amount \\
\hline Multiplex- & Positive & 0 & 0 & 0 \\
PCR test & Negative & 7 & 23 & 30 \\
& Amount & 7 & 23 & 30 \\
\hline
\end{tabular}

Users of contraceptive pills, the ranked first in this research with 18 respondents $(60.0 \%)$, followed by as many as six people $(20.0 \%)$ injection contraception users. Complete data on the distribution of respondents by contraception can be seen in Table 2. This result was consistent with several previous studies conducted by (Anindita 2006), which showed no significant correlation between the use of hormonal contraceptives with the incidence of vaginal candidiasis $(66.7 \%)$. The highestrogen oral contraceptive use increased colonization of Candida spp. in the vagina (Otasevic et al. 2002).

\section{Culture test}

Culture examination of 79 samples of vaginal secretions on SDA medium, 30 samples obtained positive with Candida infection (Table 3). After the culture with ASD medium, followed by germ tube test to distinguish albicans or non-albicans. Results of this study were higher than the prevalence of vaginal candidiasis research results $21 \%$. This can be caused because of the majority of respondents were users of oral contraceptives. According to the theory that high levels of estrogen in birth control pills are one of the causes of vaginal candidiasis (Tasic et al. 2002).

\section{Examination results fermentation and multiplex-PCR}

Identification Candida spp. was conducted using two methods, fermentation and multiplex-PCR methods, and the results are prepared in Table 4. In this study, a discrepancy was found between the test fermentation with multiplex-PCR test for some species, for example, $C$. glabrata were found in the fermentation test, on the same sample by multiplex-PCR test identified as C. krusei. In addition to $C$. albicans were identified in the fermentation test, with multiplex-PCR test on the same sample identified as $C$. stellatoidea, only one sample was identified as $C$. albicans equally well with fermentation test or test-PCR multiplex. Identification of the species $C$. krusei on the same sample for the second test method had almost $100 \%$ similarity.

Comparison of Candida tropicalis between identification fermentation test and multiplex-PCR test can be seen in Table 5. The results showed that with multiplexPCR test resulted $23.0 \%$ of the sample had an infection caused by more than one type of Candida species.

\section{Diagnostic test results of fermentation and multiplex-PCR}

Test sensitivity and specificity of multiplex-PCR to identify Candida species as the cause of candidiasis vaginalis on the study conducted on 30 samples were found positive by culture examination of Candida on SDA medium. As the gold standard test Fermentation with sweets (dextrose, maltose, sucrose, lactose, and galactose). 
C. krusei diagnostic test results on 30 respondents with fermentation test showed 12 positive samples and a total of 18 samples were negative. While multiplex-PCR test on the same sample obtained 19 samples were positive and 11 negative samples. Data from the species $C$. krusei diagnostic test can be seen in Table 6. Figure 1. above shows the $C$. glabrata with size 483 bp PCR product (sample number: 6, 63,79 and 80).

In this study, $C$. glabrata infections always coincide with other Candida species infections (mix-infection). $C$. tropicalis diagnostic test in this study with the results obtained by fermentation test five samples was positive and 25 negative samples, on the same sample by multiplexPCR test obtained five positive samples and 25 negative samples. Test data diagnostic C.tropicalis species can be seen in Table 6, while diagnostic test of $C$. tropicalis using fermentation method can be seen in Table 7 .

These results are also consistent with previous studies comparing multiplex-PCR method with commercial tools (API 20C kit), known multiplex-PCR has a sensitivity and specificity values were quite high $(100 \%)$ compared to API $20 \mathrm{C}$ Kit is its ability to identify species of $5-10 \%$ is not perfect, for example in the study of API 20C Kit could not identify $C$. glabrata and $C$. tropicalis but with multiplexPCR identified yeast species perfectly. In that study, the sample was identified as C. albicans turns with multiplexPCR identified as $C$. tropicalis. Results of the final identification with morphological analysis of culture and chrome agar show results as $C$. tropicalis. Figure 2 shows the size of the base pairs of C. tropicalis (218 bp or $199 \mathrm{bp}$ ) that the sample number 2 and 77.

Diagnostic test of $C$. albicans against 30 respondents with fermentation test showed three positive samples and a total of 27 samples were negative. Examination by multiplex-PCR test on the same sample contained only one known positive samples and 29 negative samples. Data diagnostic test results of $C$. albicans species can be seen in table $2 \times 2$ (Table 8 ).

The length of the PCR product for C. albicans was 218 bp and $110 \mathrm{bp}$, In this study by multiplex-PCR only one

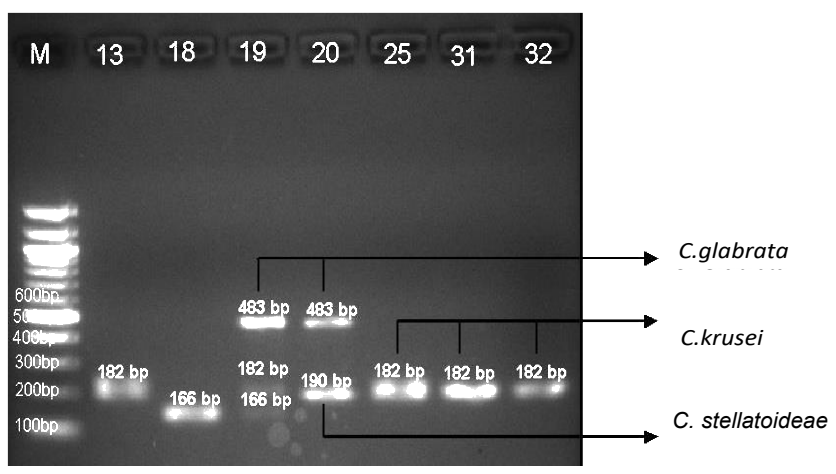

Figure 1. Samples were identified by Multiplex PCR using universal primers ITS1-ITS2 and specific primer CA3-CA4, and DNA fragment detected by agarose electrophoresis. DNA fragment bands for C. glabrata was 483 bp, C. krusei was 182 bp or $166 \mathrm{bp}$, and C. stellatoidea was $190 \mathrm{bp}$. sample was identified as C. albicans. C. stellatoidea with fermentation test in this study was found positive in three samples and 27 samples were negative. While the multiplex-PCR test was found five positive samples and 25 negative samples. C. stellatoidea diagnostic test data can be seen in Table 9.

Table 6. Diagnostic test results Candida krusei $(\mathrm{n}=30)$

\begin{tabular}{llccc}
\hline & & \multicolumn{3}{c}{ Fermentation test } \\
\cline { 3 - 5 } & & Positive & Negative & Amount \\
\hline Multiplex- & Positive & 12 & 7 & 19 \\
PCR Test & Negative & 0 & 11 & 11 \\
& Amount & 12 & 18 & 30 \\
\hline
\end{tabular}

Table 7. Diagnostic test of $C$. tropicalis $(\mathrm{n}=30)$

\begin{tabular}{llccc}
\hline & & \multicolumn{3}{c}{ Fermentation test } \\
\cline { 3 - 5 } & & Positive & Negative & Amount \\
\hline Multiplex- & Positive & 5 & 0 & 5 \\
PCR Test & Negative & 0 & 25 & 25 \\
& Amount & 5 & 25 & 30 \\
\hline
\end{tabular}

Table 8. Diagnostic test results $C$. albicans

\begin{tabular}{llccc}
\hline & & \multicolumn{3}{c}{ Fermentation test } \\
\cline { 3 - 5 } & & Positive & Negative & Amount \\
\hline Multiplex- & Positive & 1 & 0 & 1 \\
PCR Test & Negative & 2 & 27 & 29 \\
& Amount & 3 & 27 & 30 \\
\hline
\end{tabular}

Table 9. Diagnostic test results C. stellatoidea $(\mathrm{n}=30)$

\begin{tabular}{clccc}
\hline & & \multicolumn{3}{c}{ Fermentation test } \\
\cline { 3 - 5 } & & Positive & Negative & Amount \\
\hline Multiplex- & Positive & 3 & 2 & 5 \\
PCR Test & Negative & 0 & 25 & 25 \\
\hline
\end{tabular}

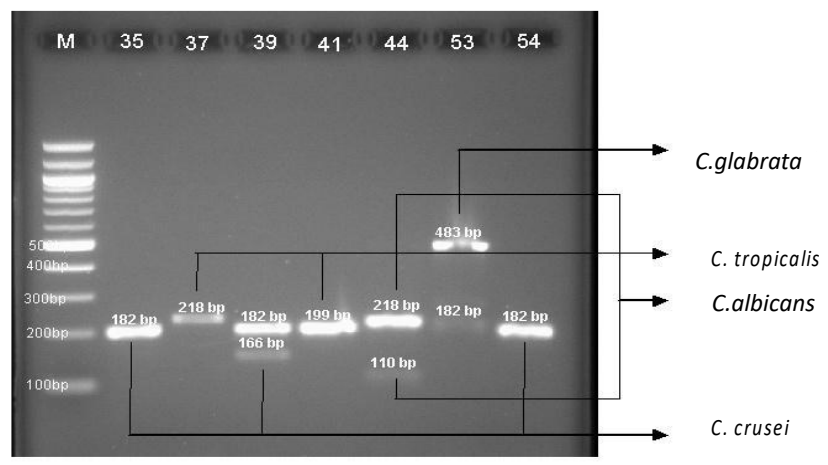

Figure 2. Samples were identified by multiplex PCR using universal primers ITS1-ITS2 and specific primer CA3-CA4, and DNA fragment detected by agarose electrophoresis. DNA fragment bands for C. glabrata was $483 \mathrm{bp}$, C. tropicalis was 218 bp or $199 \mathrm{bp}$, and C. albicans was $218 \mathrm{bp}$ and $110 \mathrm{bp}$, C. krusei was $190 \mathrm{bp}$. 
The test results of sensitivity and specificity in identifying by multiplex-PCR, C. stellatoidea have value: sensitivity $100 \%$, specificity $92.6 \%$, positive predictive value $60 \%$ and negative predictive value of $100 \%$. Large base pairs to $C$. stellatoidea with the use of a combination of universal primers ITS1-ITS2 and specific primers CA3CA4 in this study based on the literature is $190 \mathrm{bp}$ (Liguori et al. 2007). Precise and rapid identification in the case of candidiasis is must, considering each Candida species have the virulence and sensitivity to different antifungal. Based on the study of (Chang et al. 2001), known to $30.7 \%$ of $C$. glabrata isolates turns resistant against fluconazole. While the approach to the identification of Candida species with conventional techniques requires a significant financial cost and a longer time. Multiplex-PCR to identify Candida species using universal primer pairs that amplify ITS1 and ITS 2 conserve areas of $18 \mathrm{~S}$ rDNA ITS1 which covers the border, a small part of 5.8S rDNA region. Amplification of this region produces a variety of different sizes of the species Candida. In addition, this study also uses a primer that allows specific to identify several species of Candida at the same time (Chang et al. 2001).

\section{Discussion}

As previous data (Anindita 2006), the incidence of vaginal candidiasis is commonly found in women aged 2635 years $(50 \%)$. The first diagnosis of candidiasis is commonly found at the age of 17 years and $54.7 \%$ of women aged 25 years'experience vaginal candidiasis (Geiger et al. 1995). Comparison of results between test Candida species identification by fermentation and multiplex-PCR test can be seen in Table 6. Results showed that with multiplex-PCR test resulted $23.3 \%$ of the sample had an infection caused by more than one type of Candida species, it has a higher value when compared with the results of research conducted in Iran from 2006-2008 (Rad et al. 2012), in which the study showed $10.3 \%$ of respondents experiencing vaginal candidiasis caused by more than one species of Candida. Similarly, research conducted by Liguori et al (2007), identification of more than one species of Candida on the same sample by multiplex-PCR, while the routine phenotypic identification methods only identified one species and of that study concluded that the multiplex-PCR method is a test accurate in detecting Candida species, allowing for the detection of more than one species of Candida in the absence of crossreaction and without being influenced by bacteria or viruses. This can be clarified by the working principle multiplex-PCR that uses the target area ITS1 and ITS2, 5.8S combined with universal and specific multiplex-PCR primer allows up to seven Candida species identified at once in the same time. Diagnostic test for C. krusei species in this study had a sensitivity of $100 \%$, in other words, the ability of multiplex-PCR method to detect a true positive result is $100 \%$. While the value of specificity of $61.1 \%$ that it also means the ability of multiplex-PCR method in detecting true negative is $61.1 \%$. $63.2 \%$ positive predictive value (the probability of an individual suffering from vaginal candidiasis) and negative predictive value of $100 \%$ (the probability of an individual not suffering from vaginal candidiasis). Large base pairs to C. krusei with the use of a combination of universal primers ITS1-ITS2 and specific primers CA3-CA4 in this study based on the literature is 182 bp or 166 bp (Liguori et al. 2007). These results are consistent with previous studies conducted by ( $\mathrm{Rad}$ et al. 2012), multiplex-PCR has higher sensitivity and higher specificity than the API 20C in identification of Candida species for example in this study API 20C Kit could not identify both $C$. glabrata and $C$. tropicalis, while multiplex-PCR method identified perfectly. Diagnostic test for $C$. tropicalis species in this study had a sensitivity of $100 \%$, in other words, the ability of multiplex-PCR method to detect a true positive result is $100 \%$. While the value of specificity of $100 \%$, which mean that the ability multiplexPCR method in detecting true negative is $100 \%$. $100 \%$ positive predictive value (the probability of an individual suffering from vaginal candidiasis) and negative predictive value of $100 \%$ (the probability of an individual not suffering from vaginal candidiasis). Large base pairs for $C$. tropicalis with the use of a combination of universal primers ITS1-ITS2 and specific primers CA3-CA4 in this study based on the literature is $218 \mathrm{bp}$ or $199 \mathrm{bp}$. The value of base pairs is difficult to distinguish from C. albicans, but because in this study using a primer specific for $C$. albicans (CA3 and CA4), then to $C$. albicans was visible band at $110 \mathrm{bp}$ in addition to the $218-219$ bp or $198-199 \mathrm{bp}$ (Liguori et al. 2010). Research conducted by Liguori et al. (2007) which compares a routine examination phenotype with multiplex-PCR to identify Candida species also showed similar results to this study, the sensitivity and specificity of multiplex-PCR in identifying $C$. tropicalis higher than the examination phenotype.

In Table 8, multiplex-PCR sensitivity in the test species $C$. albicans was $33.3 \%$ (ability of multiplex-PCR to determine true positive), while the value is $100 \%$ specificity (ability multiplex-PCR to determine the true negative). The positive predictive value (the possibility of samples experiencing candidiasis vaginalis) is $100 \%$ and the negative predictive value (likelihood samples do not experience vaginal candidiasis) is $93.1 \%$. Results of diagnostic tests of a diagnostic method said is good if it has a value of sensitivity and high specificity. The results of diagnostic tests multiplex-PCR in previous studies have an average sensitivity and high specificity, as practiced by Rad et al. (2012) which showed that results for all species with multiplex-PCR test result by the same method of germ tube, production chlamydospore the CMA and the API 20C-AUX Kit. Test sensitivity and specificity multiplexPCR compared with 9 kinds of test phenotype in $C$. albicans species showed multiplex-PCR method has high sensitivity and specificity (100\%) (Liguori et al. 2007). API 20C AUX with the manual system, requires a long time for incubation but easy to use and not too expensive, in the study had a sensitivity of $86.9 \%$ and a specificity of $95 \%$. While the method vitek2 been using automatic systems and allows to identify a large number of species, but in that study had the lowest sensitivity value $(81.9 \%)$. Chromogenic media simple, fast and economical in 
identifying species of Candida, but the colors are formed on the media after storage and subculture so varied that it is difficult to interpret (Momani and Qaddoomi 2005). Previous research report stated that multiplex-PCR method is accurate in identifying, simple, fast without the use of toxic chemicals and expensive. Other molecular techniques have also been developed to identify infectious diseasecausing species, including $C$. albicans, but the method can not be used as a routine examination because of high cost and the need for skilled human resources. Actually, in some cases, species identification using biochemical test combined with routine examination phenotype was able to increase the sensitivity and specificity, but for specific purposes such differentiating $C$. albicans and $C$. dubliniensis still needed inspection molecular level, due to the characteristics of the same phenotype as $C$. albicans, $C$. dubliniensis also formed chlamydospore, germ tube and display the same natural resources. Identification to distinguish between two species was very important, because of the growing incidence of candidiasis caused by C. dubliniensis and C. dubliniensis known to be resistant to fluconazole (Lim and Lee 2002). The results in Table 9 are consistent with previous studies conducted on identification of Candida species using ITS2 genetic region, with the PCR method has high sensitivity. C. stellatoidea morphologically indistinguishable from $C$. albicans (McCullough et al. 1999). Test germ tube formation in the egg white and the test medium chlamydospore formation in cultured cornmeal Tween-BO (CMT test) was not able to distinguish the two species, although theoretically, the CMT test can differentiate Candida species based on the ability of specific pseudohyphae formation, but in some research turns pseudohyphae that form less specific. Theoretically, $C$. stellatoidea distinguished from $C$. albicans because it failed to form acid from sucrose, lighter pathogenicity, and formation chlamydospore late (Luo and Mitchell 2002). Fermentation and assimilation test can distinguish $C$. albicans and $C$. stellatoidea based on the ability of these species in the use of sugars. multiplex-PCR test in this study using specific primers for C. albicans allow a high sensitivity and specificity (Harmal et al. 2012). The image below shows the sample number 20 base pairs C. stellatoidea size (190 bp) (Figure 1). In this result, we stated that of the two types of examination $C$. krusei obtained was the most common cause of candidiasis (fermentation Test: 40\%, multiplex-PCR Test: 46.7\%). Using multiplex-PCR test showed that $23.3 \%$ of the sample had an infection caused by more than one species of Candida. multiplex-PCR diagnostic test for C. krusei has a sensitivity of $100 \%$, specificity: $61.1 \%$, NPP: $63.2 \%$ and NPN: $100 \%$, against $C$. glabrata has a value of $0 \%$ sensitivity, specificity: $100 \%$, NPP: $0 \%$ and NPN: $76.7 \%$. Against $C$. tropicalis has a sensitivity of $100 \%$, specificity: 100\%, NPP: $100 \%$ and NPN: $100 \%$, against C. albicans has a sensitivity of $33.3 \%$, specificity: $100 \%$, NPP: $100 \%$ and NPN: 93, 1\%, as well as against $C$. stellatoidea had a sensitivity of $100 \%$, specificity: $92.6 \%$, NPP: $60 \%$ and NPN: $100 \%$.

Based upon above results it is concluded that both fermentation and multiplex-PCR tests revealed C. krusei as the most common cause of candidiasis (test fermentation: 40\%, multiplex-PCR Test: 46.7\%). Using multiplex-PCR test showed that $23.3 \%$ of the sample had an infection caused by more than one species of Candida. MultiplexPCR diagnostic test for C. krusei has a sensitivity of $100 \%$, specificity: $61.1 \%$, NPP: $63.2 \%$ and NPN: $100 \%$, against C. glabrata has a value of $0 \%$ sensitivity, specificity: $100 \%$ , NPP: $0 \%$ and NPN: $76.7 \%$. Against C. tropicalis has a sensitivity of $100 \%$, specificity: $100 \%$, NPP: $100 \%$ and NPN: $100 \%$, against C. albicans has a sensitivity of $33.3 \%$, specificity: 100\%, NPP: $100 \%$ and NPN: $93.1 \%$, as well as against $C$. stellatoidea had a sensitivity of $100 \%$, specificity: $92.6 \%$, NPP: $60 \%$ and NPN: $100 \%$.

\section{ACKNOWLEDGEMENTS}

The authors would like to thank Universitas Sriwijaya, Indonesia that supported and facilitated this project. The authors declare that there is no conflict of interest.

\section{REFERENCES}

Anindita W, Martini S. 2006. Faktor risiko kejadian kandidiasis vaginalis pada akseptor KB. Indonesian J Publ Health 3: 24-28. [Indonesian]

Chang HC, Leaw SN, Huang AH, Wu TL, Chang TC. 2001. Rapid identification of yeasts in positive blood cultures by a multiplex PCR method. J Clin Microbiol 39 (10): 3466-3471.

da Silva GA, Bernardi TL, Schaker PDCD, Menegotto M, Valente P. 2012. Rapid yeast DNA extraction by boiling and freeze-thawing without using chemical reagents and DNA purification. Braz Arch Biol Technol 55 (2): 319-327.

Geiger AM, Foxman B, Gillespie BW. 1995. The epidemiology of vulvovaginal candidiasis among university students. Am J Publ Health 85 (8): 1146-1148.

Harmal NS. 2012. Simplex and triplex Polymerase Chain Reaction (PCR) for identification of three medically important Candida species. Af J Biotechnol 11 (65): 12895-12902.

Haryani M, Urip KS, Wasitaatmadja SM. 2003. Vulvovaginal candidosis caused by Candida Non-Albicans, proportion and clinical characteristics in the Dr. Cipto Mangunkusumo National General Hospital, Jakarta. Med J Indones 12: 142-147. [Indonesian]

Liguori G, Lucariello A, Colella G, De Luca A, Marinelli P. 2007. Rapid identification of Candida species in oral rinse solutions by PCR. J Clin Pathol 60 (9): 1035-1039.

Liguori G, Onofrio VDI, Gallé F, Lucariello A, Albano L, Catania MR, Guida M. 2010. Candida albicans identification: comparison among nine phenotypic systems and a multiplex PCR. J Prev Med Hyg 51 (3): 121-124.

Lim Y, Lee D. 2002. Multiplex polymerase chain reaction assay for simultaneous detection of Candida albicans and Candida dubliniensis. J Microbiol 40 (2): 146-150.

Luo G, Mitchell TG. 2002. rapid identification of pathogenic fungi directly from cultures by using multiplex PCR rapid identification of pathogenic fungi directly from cultures by using multiplex PCR. J Bacteriol 40 (8): 2860.

McCullough MJ, Clemons KV, Stevens DA. 1999. Molecular and phenotypic characterization of genotypic Candida albicans subgroups and comparison with Candida dubliniensis and Candida stellatoidea. J Clin Microbiol 37 (2): 417-421.

Momani OM, Qaddoomi A. 2005. Identification of Candida dubliniensis in a diagnostic microbiology laboratory. East Mediterr Health J 11 (3): 366-371.

Omar AA. 2001. Gram stain versus culture in the diagnosis of vulvovaginal candidiasis. East Mediterr Health J 7 (8): 925-934

Otasevic ST, Tasic Natasa M, Tasic A, Mitrovic S. 2002. Recurrent genital candidosis of woman: consequence of reinfection or relapse. Med Biol 9 (3): 217-222. 
Rad MM, Zafarghandi AS, Amel Zabihi M, Tavallaee M, Mirdamadi Y. 2012. Identification of Candida species associated with vulvovaginal candidiasis by multiplex PCR. Infect Dis Obstet Gynecol 2012: 1-5.

Sobel JD, Wiesenfeld HC, Martens M, Danna P, Hooton TM, Rompalo A, Sperling M, Livengood C $3^{\text {rd }}$, Horowitz B, Von Thron J, Edwards L, Panzer H, Chu TC. 2004. Maintenance fluconazole therapy for recurrent Vulvovaginal candidiasis. N Eng J Med 351 (9): 876-883.
Tarini NMA, Wahid IMH, Ibrahim F, Yasmon A, Djauzi S. 2010. Development of multiplex-PCR assay for rapid detection of Candida spp. Med J Indones 19 (2): 83-88. [Indonesian]

Wahyuningsih R, Eljannah SM, Mulyati. 2012. Identifikasi Candida spp. dengan medium kromogenik. J Indon Med Assoc 62 (3): 83-89. [Indonesian] 